13

\title{
Влияние энергии ионной стимуляции на удельное электросопротивление углеродных пленок, полученных методом импульсно-плазменного осаждения в атмосфере азота
}

\author{
(С) И.А. Завидовский, О.А. Стрелецкий, О.Ю. Нищак, А.А. Хайдаров
}

Московский государственный университет им. М.В. Ломоносова,

Москва, Россия

E-mail: ia.zavidovskii@physics.msu.ru

Поступила в Редакцию 26 марта 2019 г.

В окончательной редакции 2 июля 2019 г.

Принята к публикации 2 июля 2019 г.

Исследованы тонкие углеродные пленки, полученные методом импульсно-плазменного ионностимулированного распыления графита в атмосфере смеси аргона и азота. Результаты спектроскопии характеристических потерь энергии электронов и электронной дифракции свидетельствуют о росте графитовой составляющей при увеличении энергии ионной стимуляции. Применение ионной стимуляции в процессе напыления пленок позволяет управлять их удельным сопротивлением, изменяя его от $10^{5}$ до $10^{2} \Omega \cdot \mathrm{cm}$.

Ключевые слова: ионно-плазменное осаждение, тонкие пленки, аморфный углерод, просвечивающая электронная микроскопия, спектроскопия характеристических потерь энергии электронов, удельное сопротивление.

DOI: 10.21883/FTT.2019.11.48436.435

\section{1. Введение}

Углеродные пленки вызывают интерес исследователей благодаря возможности их применения в различных областях науки и техники, например, углеродные покрытия могут быть использованы для уменьшения трения [1], создания гидрофобной поверхности [2], улучшения биосовместимости медицинских изделий [3] и в других приложениях.

Благодаря способности углерода находиться в состоянии с различной гибридизацией $\left(s p^{1}, s p^{2}\right.$ и $s p^{3}$, соответствующие структуре карбина, графита и алмаза), свойства углеродных материалов в значительной степени зависят от их фазового состава: доли различных связей [4], наличия субструкур в составе образца $[5,6]$. Изменение условий осаждения (рабочая атмосфера, мощность разряда, стимуляция ионами, подача смещения на подложку и др.) позволяет модифицировать структуру пленок и подобрать режим, в котором покрытие будет обладать желаемыми характеристиками. Таким образом, создание тонких углеродных пленок, оптимальных для использования в конкретном направлении, требует детального анализа структуры образцов, полученных при различных параметрах напыления.

Возможность использования углеродных пленок в различных электрофизических приложениях обусловлена тем, что их удельное электросопротивление может изменяться в диапазоне от $10^{2}$ до $10^{14} \Omega \cdot \mathrm{cm}$ [7]. В то же время, допирование углеродных пленок атомами различных металлов может уменьшить эту величину до $10^{-5} \Omega \cdot \mathrm{cm}$. Нанесение допированных углеродных пленок может осуществляться различными методами: с помощью сочетания процесса химического осаждения в газовой фазе и распыления металлической мишени [8], высокочастотным магнетронным распылением мишеней карбидов [9,10], совместным распылением графитовой и металлической магнетронных мишеней [11]. Однако недостатком допирования металлами может являться ухудшение коэффициента износа [8], критической нагрузки и твердости [10] или увеличение коэффициента трения таких покрытий [9].

Изменение электропроводности углеродных пленок возможно также при помощи внедрения в их структуру других донорных и акцепторных атомов [12-14].

Особый интерес, возросший в связи с развитием технологий анализа поверхности [15], представляет модификация углеродных покрытий при помощи азота. Современные методы анализа позволяют установить вклад азота в формирование фаз с $s p^{2}$ и $s p^{3}$-гибридизацией, который может приводить к существенному преобразованию структуры пленки и изменению ее электрофизических свойств [16,17].

Азотированные углеродные пленки находят свое применение в биологической визуализации, солнечной энергетике, в качестве функциональных покрытий и др. [18-20].

В настоящей работе приводится исследование тонких углеродных пленок, полученных методом импульсноплазменного осаждения в атмосфере смеси аргона и азота при различных энергиях стимуляции ионным пучком. Структурные свойства полученных углеродных пленок были исследованы методами просвечивающей электронной микроскопии (ПЭМ) и спектроскопии характеристических потерь энергии электронов (СХПЭЭ). Удельное сопротивление пленок измерялось четырехзондовым методом. 


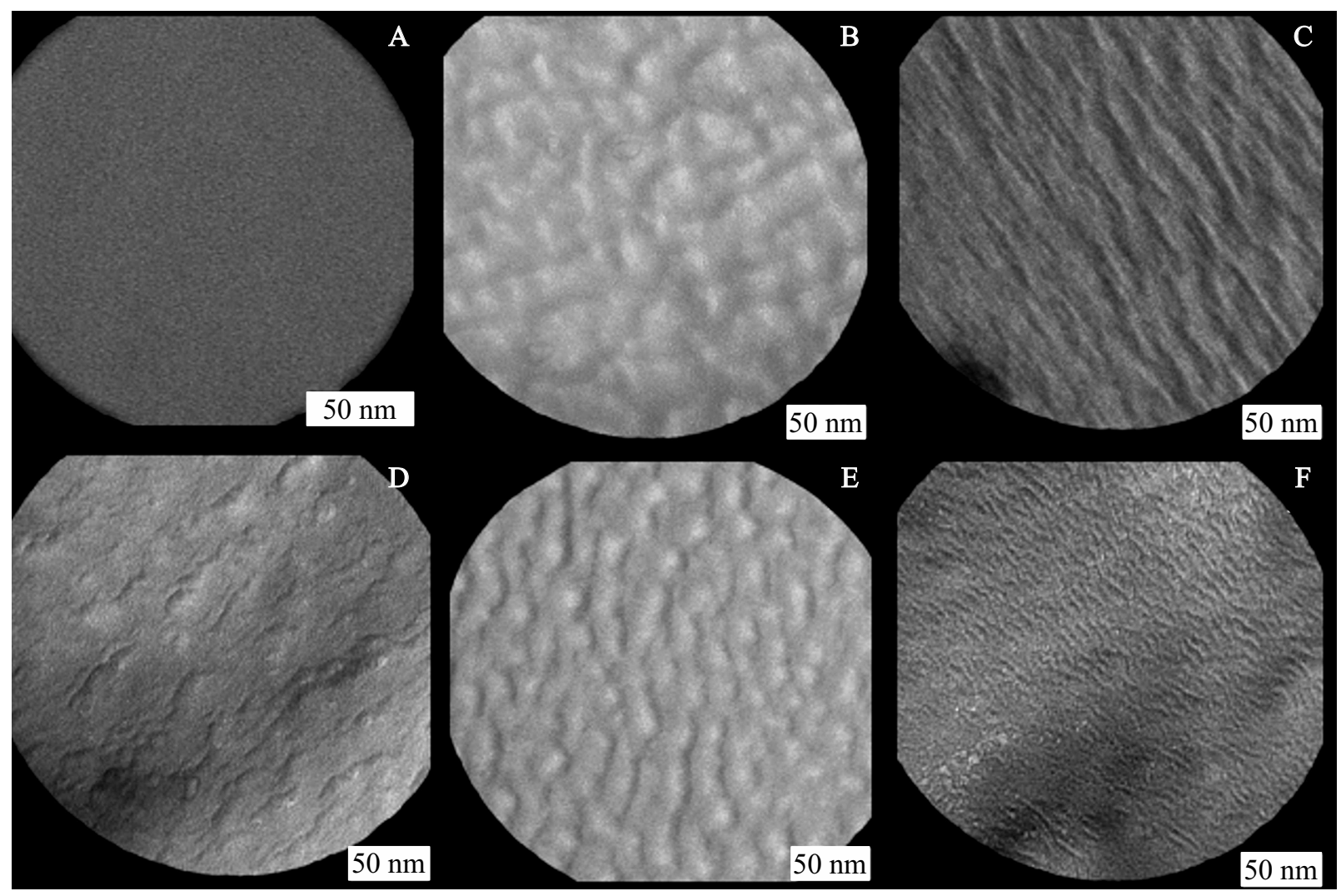

Рис. 1. Текстура образцов в зависимости от энергии стимуляции (без стимуляции $-A, 200 \mathrm{eV}-B, 300 \mathrm{eV}-C, 400 \mathrm{eV}-D$, $600 \mathrm{eV}-E, 800 \mathrm{eV}-F)$.

\section{2. Получение образцов и методы их исследования}

Исследуемые образцы изготавливались методом импульсно-плазменного ионно-стимулированного распыления графита. Напыление осуществлялось в атмосфере смеси аргона и азота при давлении $10^{-3}$ Torr. Относительная концентрация азота в рабочей атмосфере не изменялась и составляла $25 \%$. Время импульса разряда составляло порядка $1 \mathrm{~ms}$, частота следования импульсов - $1 \mathrm{~Hz}$, мощность разряда $-1 \mathrm{~kW}$. В качестве подложек использовались кремниевые и титановые пластины, а также кристаллы поваренной соли. Температура подложек во время процесса осаждения не превышала $50^{\circ} \mathrm{C}$ В ходе процесса напыления при помощи холловского ионного источника осуществлялась стимуляция роста пленок ионами азота и аргона различных энергий (от 200 до $700 \mathrm{eV}$ ) и фиксированном токе. Контроль толщины в ходе напыления был реализован при помощи пьезоэлектрического датчика. Толщина всех образцов составляла порядка $50 \mathrm{~nm}$.

Образцы были исследованы методами просвечивающей электронной микроскопии, электронной дифракции и спектроскопии характеристических потерь энергии электронов при помощи комплекса LEO 912 AB с ускоряющим напряжением $120 \mathrm{keV}$.
Удельное сопротивление пленок было измерено четырехзондовым методом. Измерения были проведены с помощью системы Jandel RMS-EL-Z.

\section{3. Экспериментальные результаты}

\section{1. Текстура образцов}

Изображения структуры пленок, полученные при помощи ПЭМ, приведены на рис. 1.

Все образцы, кроме нанесенного без ионной стимуляции, имеют неоднородную текстуру, изменяющуюся в зависимости от энергии ассистирующих ионов.

В работах $[21,22]$ для углеродных пленок наблюдалось образование схожего рельефа. Причиной его возникновения являлась ионная стимуляция или обработка структур ионным пучком, направленным, как и в нашем случае, под углом к поверхности пленки.

\section{2. Удельное электросопротивление пленок}

Изменение удельного сопротивления углеродных пленок в зависимости от энергии стимуляции представлено на рис. 2.

Ионная стимуляция приводит к значительному уменьшению удельного электросопротивления исследуемых 


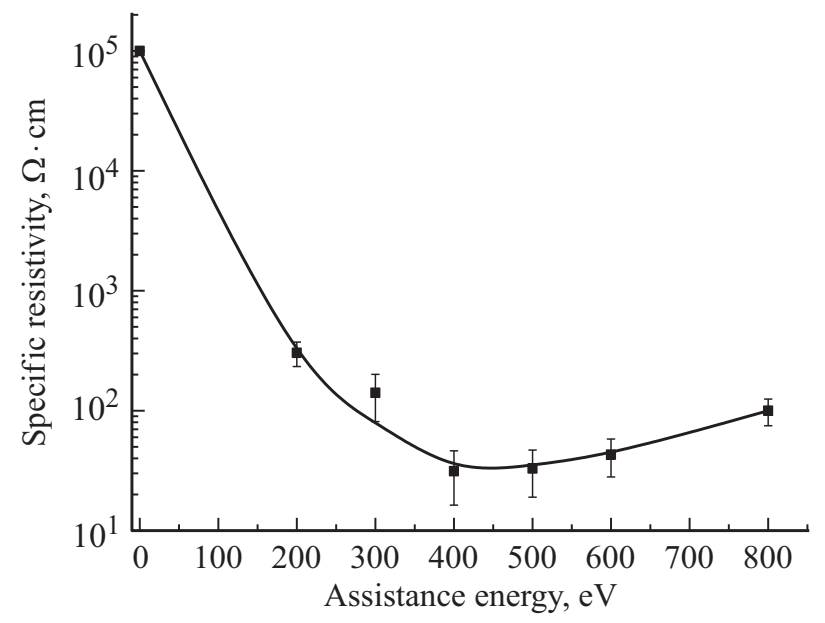

Рис. 2. Зависимость удельного электросопротивления образцов от энергии ионной стимуляции.

образцов. При этом для углеродной пленки, нанесенной при энергии стимулирующих ионов $400 \mathrm{eV}$, наблюдается минимум сопротивления. Минимальное значение удельного сопротивления составляет $30 \Omega \cdot \mathrm{cm}$.

Для объяснения зависимости электросопротивления исследуемых образцов от энергии стимуляции были проведены исследования их структуры.

\section{3. Электронная дифракция}

На рис. 3 представлены результаты фотометрирования дифракционных картин образцов, нанесенных при различной энергии стимуляции.

Дифракционная картина образца, напыленного без ионной стимуляции, характерна для аморфных углеродных структур [23-25]. На ней наблюдается два широких максимума с межплоскостными расстояниями $2.06 \AA$ и $1.15 \AA$. Линия $2.06 \AA$ характерна для аморфных алмазоподобных пленок, ее сильное уширение характеризует большую степень разупорядоченности структуры.

При введении ионной стимуляции в процесс напыления на дифракционных картинах полученных образцов появляется дополнительная линия вблизи $3.35 \AA$, положение которой соответствует межплоскостному расстоянию в графите [23]. С ростом энергии стимулирующих ионов происходит увеличение относительной интенсивности данной линии, которое связано с ростом доли графитовой компоненты в структуре пленки. Таким образом, ионная стимуляция в атмосфере азота приводит к появлению в структуре пленок графитовых нанокластеров, влияющих на их электрофизические свойства.

\section{4. Спектроскопия характеристических потерь энергии электронов}

Для более детального анализа изменения структуры образцов были исследованы спектры, отвечающие потерям на возбуждение электронов с остовного уровня углерода. Вычитание фона было проведено при помощи метода Тугарда [26,27]. При этом полученные спектры (рис. 4) имеют два характерных максимума, отвечающих переходу электронов с К-оболочки атома углерода на уровни энергии, соответствующие $\pi^{*}$ - и $\sigma^{*}$-подзонам.

Для спектров характеристических потерь энергии электронов интенсивность данных линий свидетельствует о количестве $\pi$ - и $\sigma$-связей [28]. Согласно [29], $\pi^{*}$-пик в районе низких энергий можно аппроксимировать гауссовой составляющей, а отношение ее площади к площади $\sigma^{*}$-пика пропорционально соотношению $\pi$ - и $\sigma$-связей в структуре. Данное соотношение для исследуемых образцов в зависимости от энергии стимуляции приведено на рис. 5 .

Полученные данные свидетельствуют о росте интенсивности линии, отвечающей переходу электронов на свободные $\pi$-орбитали по отношению к линии, соответствующей переходу на $\sigma$-орбитали атома углерода. Увеличение данного параметра для пленок аморфного углерода соответствует возрастанию

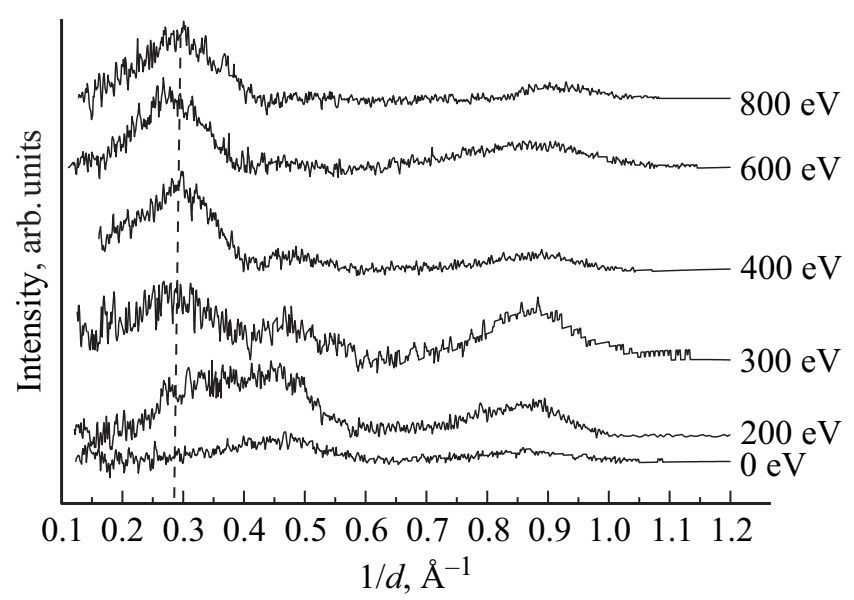

Рис. 3. Дифрактограммы пленок, изготовленных в атмосфере смеси аргона и азота при различной энергии стимуляции.

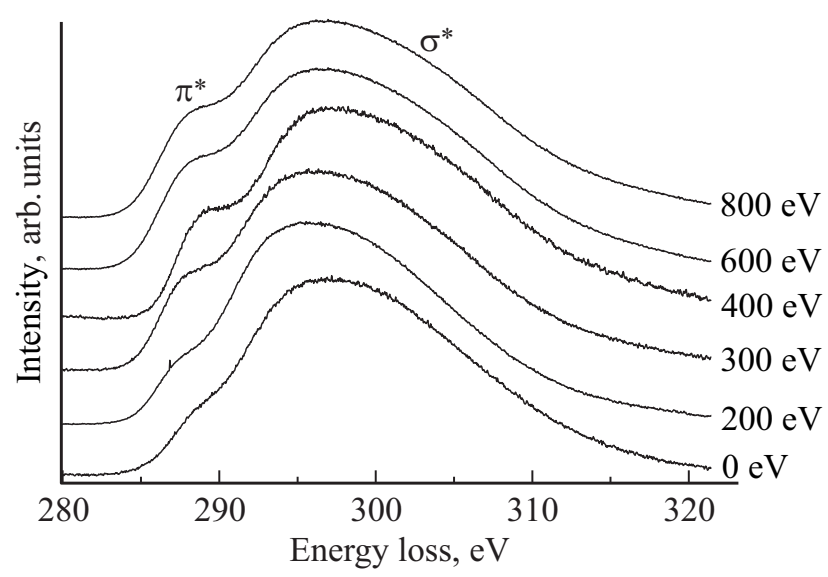

Рис. 4. Спектры характеристических потерь энергии электронов с остовного уровня углерода. 


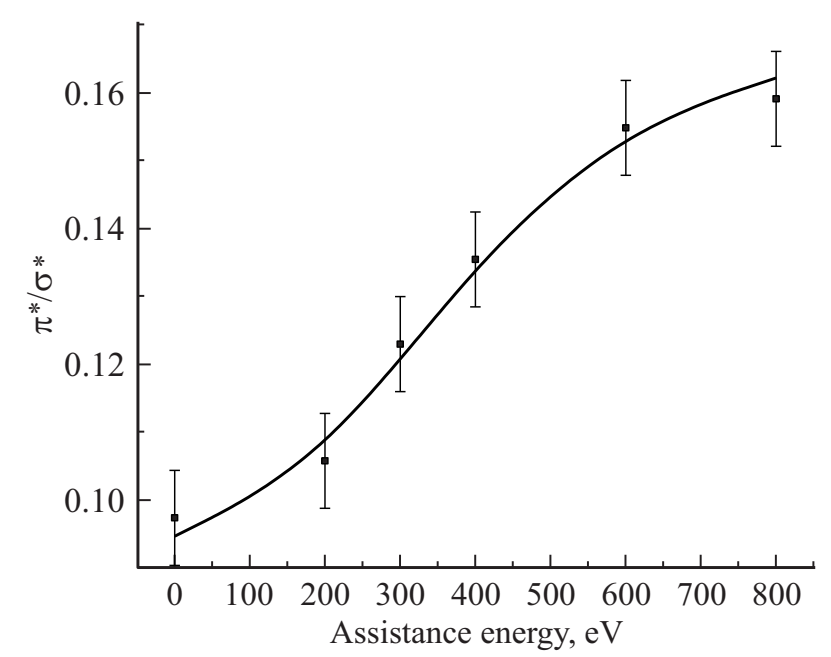

Рис. 5. Зависимость отношения интенсивностей $\pi^{*}$ и $\sigma^{*}$-пиков СХПЭЭ.

доли $s p^{2}$-гибридизованных атомов по отношению к $s p^{3}$-гибридизованным атомам в структуре пленки [30].

Данная зависимость, коррелирующая с данными электронной дифракции, свидетельствует о росте графитовой компоненты при увеличении энергии стимулирующих ионов.

\section{5. Особенности проводимости пленок}

Таким образом, введение ионного ассистирования в процесс напыления приводит к эффективному встраиванию азота в структуру, уменьшающему электросопротивление с $10^{5}$ до $10^{2} \Omega \cdot \mathrm{cm}$. Данные электронной дифракции позволяют связать это с образованием в углеродной пленке компоненты с низким электросопротивлением - графитовых нанокластеров.

В работе [31] показано, что на сопротивление двухкомпонентных композитов влияет как сопротивление их составляющих, так и их соотношение. Однако полученная в настоящей работе зависимость не позволяет полностью описать проводимость полученных нами образцов, поскольку не учитывает явления рассеяния, которые происходят на границе раздела фаз и дают, согласно [32], существенный вклад в сопротивление тонких пленок. Уменьшение размера кристаллитов, как правило, происходящее при увеличении энергии стимулирующих ионов, увеличивает поверхность границы раздела фаз и приводит к возрастанию электросопротивления [33,34].

Исходя из вышесказанного, при образовании графитовых нанокластеров в условиях ионной стимуляции происходит протекание двух конкурирующих процессов: с одной стороны, с ростом энергии стимуляции увеличивается доля графитовой фазы в структуре пленок, а с другой - уменьшается размер графитовых кластеров, что и обуславливает появление минимума сопротивления пленок при энергии $400 \mathrm{eV}$.

\section{4. Заключение}

В работе представлены результаты исследования тонких углеродных пленок, полученных в атмосфере смеси аргона и азота методом импульсно-плазменного ионноассистированного распыления графита. Удельное электросопротивление пленок составило $10-10^{2} \Omega \cdot \mathrm{cm}$, что на 3-4 порядка меньше сопротивления пленок, изготовленных без ионной стимуляции. Ионная стимуляция приводит к образованию графитовых нанокластеров, доля которых возрастает с увеличением энергии ассистирования. При энергии стимуляции $400 \mathrm{eV}$ наблюдается минимум удельного электросопротивления, что можно объяснить одновременным протеканием двух конкурирующих процессов: увеличением доли графитовой фазы в структуре пленок и уменьшением размера графитовых кластеров с ростом энергии стимуляции.

\section{Конфликт интересов}

Авторы заявляют, что у них нет конфликта интересов.

\section{Список литературы}

[1] D. He, S. Zheng, J. Pu, G. Zhang, L. Hu. Tribology Int. 82, 20 (2015). DOI: 10.1016/j.triboint.2014.09.017

[2] R. Rincón, A. Hendaoui, J. de Matos, M. Chaker. J. Appl. Phys. 119 22, 223303 (2016). DOI: 10.1063/1.4953684

[3] T.T. Liao, T.F. Zhang, S.S. Li, Q.Y. Deng, B.J. Wu, Y.Z. Zhang, Y.J. Zhou, Y.B. Guo, Y.X. Leng, N. Huang. Mater. Sci. Eng. C. 69, 75 (2016). DOI: 10.1016/j.msec.2016.07.064

[4] R. Paul, S.N. Das, S. Dalui, R.N. Gayen, R.K. Roy, R. Bhar, A.K. Pal. J. Phys. D 41 5, 055309 (2008). DOI: $10.1088 / 0022-3727 / 41 / 5 / 055309$

[5] A. Varma, V. Palshin, E.I. Meletis. Surface Coatings Technology 148 2-3, 305 (2001). DOI: $10.1016 / \mathrm{s} 0257-8972(01) 01350-0$

[6] A. Hu, I. Alkhesho, H. Zhou, W.W. Duley. Diamond Related Mater. 16 1, 149 (2007). DOI: 10.1016/j.diamond.2006.04.008

[7] A. Grill, V. Patel, S. Cohen. Diamond Related Mater. 3, 281 (1994).

[8] H. Dimigen, H. Hübsch, R. Memming. Appl. Phys. Lett. 50, 1056 (1987).

[9] M. Wang, K. Schmidt, K. Reichelt. J. Mater. Res. 7, 6 (1992).

[10] C. Bauer, H. Leiste, M. Stüber, S. Ulrich, H. Holleck. Diamond Related Mater. 11 3-6, 1139 (2018). DOI: $10.1016 / \mathrm{s} 0925-9635(01) 00714-2$

[11] C.P. Klages, R. Memming. Mater. Sci. Forum 52-53, 609 (1989).

[12] S. Liza, J. Hieda, H. Akasaka, Ohtake, N., Tsutsumi, Y., Nagai, A., T. Hanawa. Sci. Technology Adv. Mater. 18 1, 76 (2017). DOI: 10.1080/14686996.2016.1262196

[13] R. Dey, S. Dolai, S. Hussain, R. Bhar, A.K. Pal. Diamond and Related Materials, 82, 70 (2018). DOI: 10.1016/j.diamond.2018.01.002

[14] J. Lanigan, H.M. Freeman, C. Wang, M.B. Ward, A. Morina, A. Neville, R. Brydson. RSC Adv. 7 69, 43600 (2017). DOI: $10.1039 / \mathrm{c} 7 \mathrm{ra08959g}$ 
[15] L. Perini, C. Durante, M. Favaro, V. Perazzolo, S. Agnoli, O. Schneider, G. Granozzi, A. Gennaro. ACS Appl. Mater. Interfaces 7 2, 1170 (2015). DOI: 10.1021/am506916y

[16] W. Zhang, Y. Xia, J. Ju, L. Wang, Z. Fang, M. Zhang. Solid State Commun. 126 3, 163 (2003). DOI: $10.1016 / \mathrm{s} 0038-1098(02) 00673-7$

[17] B.S. Satyanarayana, A. Hart, W.I. Milne, J. Robertson. Diamond Related Mater. 7 2-5, 656 (1998). DOI: $10.1016 / \mathrm{s} 0925-9635(97) 00296-3$

[18] J. Liu, H. Wang, M. Antonietti. Chem. Soc. Rev. 45, 2308 (2016).

[19] L. Jia, H. Wang, D. Dhawale, C. Anand, M.A. Wahab, Q. Ji, K. Arigab, A. Vinuab. Chem. Commun. 50 45, 5976 (2014). DOI: $10.1039 / \mathrm{c} 4 \mathrm{cc} 02042 \mathrm{a}$

[20] E. Cutiongco, D. Li, Y. Chung. J. Tribology 118, 543 (1996).

[21] L.K. Cheah, X. Shi, J.R. Shi, E.J. Liu, S.R.P. Silva. J. NonCryst. Solids 242 1, 40 (1998). DOI: 10.1016/s0022-3093(98)00787-x

[22] A. Stanishevsky. Thin Solid Films, 398-399, 560 (2001). DOI: 10.1016/s0040-6090(01)01318-9

[23] Q. Wang, C. Wang, Z. Wang, J. Zhang, D. He. Appl. Phys. Lett. 91 14, 141902 (2007). DOI: 10.1063/1.2794017

[24] A.A. Voevodin, J.G. Jones, J.S. Zabinski, Z. Czigány, L. Hultman. J. Appl. Phys. 92 9, 4980 (2002). DOI: $10.1063 / 1.1509106$

[25] J. Neidhardt, L. Hultman, Z. Czigány. Carbon. 42, 2729 (2004). DOI: 10.1016/j.carbon.2004.06.011

[26] S. Tougaard. Surf. Sci. 464 2-3, 233 (2000). DOI: $10.1016 / \mathrm{s} 0039-6028(00) 00684-1$

[27] S. Tougaard. Surf. Sci. 216 3, 343 (1989). DOI: $10.1016 / 0039-6028(89) 90380-4$

[28] J. Kulik, G.D. Lempert, E. Grossman, D. Marton, J.W. Rabalais, Y. Lifshitz. Phys. Rev. B 52 22, 15812 (1995). DOI: $10.1103 /$ physrevb.52.15812

[29] A.C. Ferrari, A. Libassi, B.K. Tanner, V. Stolojan, J. Yuan, L.M. Brown, S.E. Rodil, B. Kleinsorge, J. Robertson. Phys. Rev. B 62 16, 11089 (2000). DOI: 10.1103/physrevb.62.11089

[30] D.L. Pappas, K.L. Saenger, J. Bruley, W. Krakow, J.J. Cuomo, T. Gu, R.W. Collins. J. Appl. Phys. 7111, 5675 (1992). DOI: $10.1063 / 1.350501$

[31] D.S. McLachlan, M. Blaszkiewicz, R.E. Newnham. J. Am. Ceram. Soc. 73 8, 2187 (1990) DOI: $10.1111 /$ j.1151-2916.1990.tb07576.x

[32] A.F. Mayadas, M. Shatzkes. Phys. Rev. B 1 4, 1382 (1970). DOI: $10.1103 /$ physrevb.1.1382

[33] R.A. Roy, J.J. Cuomo, D.S. Yee. J. Vac. Sci. Technology A 63 , 1621 (1988). DOI: $10.1116 / 1.575339$,

[34] J.J. Cuomo, S.M. Rossnagel, H.R. Kaufman. Handbook of Ion Beam Processing Technology: Principles, Deposition, Film Modification and Synthesis. Noyes Publications, Westwood, New Jersey, USA. (1989). 457 p. ISBN 0-8155-1199-X

Редактор К.В. Емиев 